\title{
[editorial]
}

\section{Sobre los efectos adversos en la vida real}

\author{
Darío Scublinsky \\ Médico Reumatólogo. \\ Coordinador de la Unidad de Farmacovigilancia, Primera Cátedra de Farmacología, Facultad de Medicina, UBA, Nodo ANMAT.
}

En los tiempos en que la terapéutica farmacológica estaba en sus inicios, allá por fines del siglo XIX, se publica en The Lancet uno de los primeros reportes científicos de efectos adversos, que se trataba de las drogas anestésicas utilizadas en dicho momento ${ }^{1}$. Algunos años después, la American Medical Association publica un artículo donde se denuncian los efectos mortales del jarabe de Winslow, "capaz de calmar a cualquier persona o animal", que era ni más ni menos que una combinación de morfina y alcohol ${ }^{2}$. Con el inicio de la farmacología moderna se comienzan a describir ciertos efectos deletéreos de los primeros antibióticos, como las sulfamidas y la penicilina, así como de la aspirina. Sin embargo, lejos están los reportes de estas primeras drogas respecto a las tragedias del dietilenglicol y la talidomida: dietilenglicol, un solvente industrial de gusto dulzón con propiedades tóxicas conocidas desde $1930^{3}$, fue utilizado cantidad de veces en la historia de la Medicina del siglo XX acompañando a drogas como sulfas, soluciones antitusígenas, jarabes de paracetamol y otros tantos, dando lugar a miles de muertos, incluyendo seis víctimas en la ciudad de La Plata en 1992 (caso Propóleo Laboratorio Huilén $)^{4,5}$.

La tragedia de la talidomida marca el inicio de la farmacovigilancia moderna. Recordemos que esta droga fue comercializada como sedante de venta libre en Alemania en 1957. Muy pronto fue comercializada en 45 países incluyendo la Argentina. En Estados Unidos, una joven farmacóloga que comenzaba a trabajar en la FDA, pidió estudios de seguridad al laboratorio fabricante, que nunca los recibió; eso motivó que la droga nunca fuera aprobada en ese país. En 1958 comenzó a ser prescripta en el resto del mundo para combatir los vómitos y el malestar matutino del primer trimestre del embarazo, a pesar de que nunca se habían realizado estudios de teratogenicidad. Fue la droga más vendida después de la aspirina, y para 1960 ya había 15.000 casos de focomelia (miembros de aletas de foca) y otras malformaciones congénitas que llevarían a la muerte al $40 \%$ de los afectados en su primer año de vida ${ }^{6}$. En 1961 un obstetra australiano y un pediatra alemán alertaban sobre una epidemia de casos de focomelia, ${ }^{7,8}$. En diciembre de 1961, luego de mayor evidencia científica y sobre todo de un artículo en un periódico alemán que vinculaba directamente los casos de malformaciones a la talidomida, el laboratorio Gruenenthal retiraba la droga de la venta. Paralelamente, la OMS prohibía el uso de la talidomida. En 1963, la OMS adoptó una resolución que reafirmaba la necesidad de un rápido reporte de los efectos indeseados que surgieran con los medicamentos. $Y$ en 1964 nace finalmente la farmacovigilancia moderna en Gran Bretaña, con las fichas amarillas ("Yellow Card"), como sistema de notificación espontánea o voluntaria".

La OMS define la Farmacovigilancia (FV) como la ciencia y las actividades relativas a la detección, evaluación, comprensión y prevención de los efectos adversos de los medicamentos o cualquier otro problema de salud relacionado con ellos. Para cumplir con dicho propósito se proponen objetivos vinculados al fomento del uso de medicamentos de manera racional y segura entre los efectores de la salud, así como una adecuada comunicación en general, para mejorar la salud pública, prevenir los daños y maximizar los beneficios de los medicamentos ${ }^{10}$.

$\mathrm{La}$ aparición de los ensayos clínicos (EC) ha cambiado dramáticamente la posibilidad de la llegada de medicamentos inseguros a la población. Sin embargo, los EC tienen ciertas dificultades en la detección de efectos adversos:

1) Los EC suelen excluir a las embarazadas, a los niños, ancianos y a individuos con co-morbilidades.

2) El tamaño de la muestra siempre resulta reducido como para detectar efectos adversos de baja frecuencia de presentación.

3) Los EC tienen duración limitada, por lo que no se pueden detectar efectos adversos tardíos (ej., cáncer).

\section{Correspondencia}

E-mail: darioscublinsky@yahoo.com.ar 
4) En el EC, las variables se encuentran controladas, los pacientes son más homogéneos que en el hospital o el consultorio. En la "vida real" suele haber medicamentos que se usan en forma concomitante (algunos no previstos en el estudio y con posibles interacciones), condiciones climáticas, geográficas, sociales, culturales y nutricionales diferentes, que hacen que el tratamiento se vea modificado y suelan aparecer otros "efectos" distintos a los del EC.

Es así que hoy en día contamos con varias modalidades de la farmacovigilancia, cada una con sus virtudes y defectos.

a) La farmacovigilancia reactiva se basa en los reportes espontáneos de los efectos adversos de los medicamentos, como los del Dr. McBride con la talidomida ${ }^{7}$. Este tipo de farmacovigilancia es la que promueve ANMAT a través de su centro de referencia y sus nodos de reporte (ej., Departamento de Farmacología de la Facultad de Medicina, UBA). Esta forma de FV es la que detecta las señales mundiales en la OMS, y procede en consecuencia.

b) La farmacovigilancia pro-activa es la que va a buscar los efectos adversos. Generalmente lo hace a través de registros (ej., BIOBADASAR), donde se pueden estudiar los casos, o los casos con controles, sin llegar a ser un estudio de casos y controles, y donde el seguimiento lo hace más parecido a los estudios de cohortes prospectivas.

c) La farmacovigilancia dirigida parte de la hipótesis de que el fármaco en cuestión podría generar efectos adversos específicos y debe ser vigilado de cerca. Por ejemplo, fue el caso del rofecoxib a principios de los 2000 , en que se estudió el riesgo $\mathrm{CV}$ inherente al uso de este inhibidor COX2 específico.

d) Más allá de estos tipos de farmacovigilancia existen los estudios epidemiológicos de efectos adversos y los ensayos clínicos (ya nombrado), que aportan otro tipo de información.

El auge de los productos biotecnológicos en Reumatología en los últimos 15 años ha llevado a un extenso control por parte de autoridades regulatorias y sociedades médicas, basado en la potencialidad de aparición de efectos adversos vinculados al sistema inmune, además de otros. Algunas sociedades médicas con gran experiencia en el uso de estos medicamentos han tomado con gran responsabilidad el control de los medicamentos que prescriben sus integrantes. En el caso de algunos países europeos, EE.UU. y países de Latinoamérica, se ha optado por vigilar de manera responsable pro-activa lo que ocurre en "el mundo real". Es por eso que en la década pasada se crean los registros de medicamentos biológicos que posteriormente darían lugar al conocimiento de nuevos efectos adversos y orientarían al uso racional de estas drogas.

Entre estos registros son de destacar, entre otros:

a) El registro británico, British Society for Rheumatology Biologics Register (BSRBR), donde se reportaron por primera vez diferentes tasas de incidencia de tuberculosis entre adalimumab, infliximab y etanercept ${ }^{11}$.

b) El registro alemán, $R A$ Observation of Biologic Therapy registry (RABBIT), que reportó el desarrollo de tumores en 74/4998 pacientes sin neoplasias previas, con una tasa de incidencia global de 6,0/1.000 PY (5,1/1.000 PY para los pacientes que recibieron agentes anti-TNF; 7,2/1.000 PY para pacientes en Anakinra; y 8,4/1.000 PY para pacientes que recibieron drogas modificadoras de la enfermedad convencionales (DMARDs) $)^{12}$. Datos de 1769 pacientes del RABBIT reportaron tasas de discontinuación de 46,3, 51,3 y 61,5\% con etanercept, adalimumab e infliximab, en combinación con metotrexato, respectivamente ${ }^{13}$.

c) El Registro Español de eventos adversos de Terapias Biológicas en Enfermedades Reumáticas (BIOBADASER) reportó una tasa de incidencia de $10 \%$ de tuberculosis en pacientes tratados con infliximab ${ }^{14}$. Este registro luego demostró la utilidad del screening de tuberculosis con su consecuente reducción en la incidencia de esta infección ${ }^{15}$.

d) El registro sueco (ARTIS), donde en un reporte de hace unos pocos días, al analizar el riesgo cardiovascular con el uso de agentes anti-TNF, sobre un total de 6592 pacientes-año estudiados, se detectó que el riesgo cardiovascular se igualaba al de la población general en los pacientes con AR que eran buenos respondedores al tratamiento ${ }^{16}$.

e) El registro estadounidense (CORRONA Consortium of Rheumatology Researchers of North America) también ha hecho grandes aportes, en que además del análisis de eventos cardiovasculares, infecciones y neoplasias, ha estudiado -entre otroslos alelos de riesgo para la predicción de respuesta a agentes anti-TNF ${ }^{17}$, y en sus últimas publicaciones analiza la tasa de discontinuación de los pacientes con AR en remisión ${ }^{18}$. 
Incluso estos registros son de utilidad también para detectar cambios en la calidad de vida de los pacientes. Es así que por ejemplo el registro alemán, en su último reporte, detecta que la terapia con DMARDs en la vida real mejora significativamente la calidad de vida, aún en pacientes artríticos con múltiples tratamientos fallidos; aún así determina que los pacientes con $\mathrm{AR}$ tienen un deterioro de la calidad de vida muy significativa respecto a la población general, y que las características de los pacientes son predictores más relevantes de respuesta que los DMARDs particularmente utilizados ${ }^{19}$.

Otras conclusiones que van más allá de la terapéutica implicada se pueden extraer también de registros de eventos adversos combinados con cohortes prospectivas generales. Por ejemplo, recientemente, fueron reportadas conclusiones de los grupos Better Anti-Rheumatic Farmaco-Therapy cohort (BARFOT), Norfolk Arthritis Register (NOAR) y la cohorte Leiden Early Arthritis Clinic (EAC), en que concluyen en una publicación conjunta luego del seguimiento de 26.300 personas/año, que la presencia de autoanticuerpos en pacientes con AR se asocia con una elevada tasa global de mortalidad. En particular, para los ACCP se asocia un aumento de la mortalidad cardiovascular, y para el factor reumatoideo, muerte vinculada a neoplasias e infecciones respiratorias ${ }^{20}$.

En Latinoamérica, la necesidad de contar con este tipo de registros se basa en disponer de datos propios de acuerdo a las modalidades de tratamiento y a las características particulares étnicas y socioculturales. Uno de los trabajos más importantes de la región es la iniciativa de la Sociedad Argentina de Reumatología, que llevó al desarrollo del registro BIOBADASAR, el cual toma de modelo al registro español BIOBADASER. Luego de años de esfuerzo, capacitación y consenso de los investigadores participantes, se llega al reporte actual de este grupo de trabajo argentino.

El primer reporte fue llevado a cabo en 2011, donde se analizaron 966 pacientes (543 casos y 423 controles), que recibieron 1132 ciclos de tratamientos con agentes biológicos en 56 centros de salud entre el $1^{\circ}$ de agosto de 2010 y el $1^{\circ}$ de abril de $2011^{21,22}$.

A diferencia de registros similares, el registro argentino también incluye pacientes tratados con medicación no biológica, que lo torna sumamente interesante para obtener conclusiones. Por otro lado, la diversidad en posibilidades de prescripción en Argentina torna el estudio más preciso que otros registros latinoamericanos o europeos donde a veces predomina ampliamente el uso de un biológico sobre los otros.

El reporte actual del registro BIOBADASAR (de la
Vega $\mathrm{M}$ y cols.) es el quinto reporte del grupo de trabajo. En el mismo se incluyeron los datos de pacientes de $\mathbf{5 2}$ centros de reumatología de Argentina ingresados desde el $1^{\circ}$ agosto de 2010 hasta el 20 agosto de 2015.

De acuerdo al reporte, las tasas de discontinuación de los biológicos son muy similares a las de España y Brasil pero con características propias. $\mathrm{Y}$ los eventos adversos infecciosos fueron los más frecuentes (cutáneos y respiratorios), en coincidencia con la bibliografía internacional.

Más allá de ciertas limitaciones en cuanto al reporte de la mortalidad y los hazard ratios ajustados para los datos de infecciones y tumores, el trabajo de BIOBADASAR es de gran aporte para el conocimiento de los eventos adversos que presentan los pacientes reumáticos bajo tratamiento con agentes biológicos en Argentina, y permite obtener conclusiones útiles para la práctica clínica.

Es de gran relevancia para la clínica reumatológica contar con la información que brinda en la actualidad este tipo de registros y que adquirirá mayor importancia a medida que pase el tiempo, se incluya mayor cantidad de pacientes/eventos y se logre dilucidar lo que ocurre con poblaciones más vulnerables que reciben estos medicamentos (ancianos, niños, embarazadas) así como el riesgo de presentar eventos adversos de aparición tardía (ej., neoplasias), y aclarar los motivos de diferencias a nivel geográfico/demográficas que se presentan en algunos registros respecto a otros.

También sería de gran utilidad la implementación de este tipo de registros en países de Latinoamérica que aún no han desarrollado esta modalidad de farmacovigilancia, para que de esa manera podamos contar con medicamentos más seguros y su uso sea totalmente racional.

\section{Bibliografía}

1. Commission on Anaesthetics. Lancet. 1893; i:629638.

2. Christen AG1, Christen JA. Sozodont powder dentifrice and Mrs. Winslow's Soothing Syrup: dental nostrums. J Hist Dent. 2000 Nov; 48(3):99105.

3. Von Oettingen WF, Jirouch EA. The pharmacology of ethylene glycol and some of its derivatives in relation to their chemical constitution and physical chemical properties. J Pharmacol Exp Ther July 1931; 42:355-372.

4. Fallo de la Sala Tercera de la Cámara Federal de 
Apelaciones de La Plata, expediente N. 12.262/05.

5. P. 681. XLIII. Fallo de la Corte Suprema de la Nación carátula: "Parisi de Frezzini, Francisca c/ Laboratorios Huilén y otros s/daños y perjuicios." -20 de octubre de 2009.

6. Takumi I, Hideki A, et al. Identification of a Primary Target of Thalidomide Teratogenicity. Science 12 Mar 2010; Vol. 327, 5971: 1345-1350.

7. McBride WG. Thalidomide and congenital abnormalities. The Lancet Vol 278, 7216, 16 Dec 1961: 1358.

8. Lenz W. The history of thalidomide. The Thalidomide Victims Association of Canada. http:// www.thalidomide.ca/history-of-thalidomide/

9. Inman WHW. Monitoring for Drug Safety. J B Lippincott Co \& MTP Press Ltd, Lancaster, 1980.

10. Organización mundial de la Salud. http://apps.who. int/

11. Dixon WG, Hyrich KL, Watson KD, Lunt M, Galloway J, Ustianowski A, et al. Drug-specific risk of tuberculosis in patients with rheumatoid arthritis treated with anti-TNF therapy: results from the British society for rheumatology biologics register (BSRBR). Ann Rheum Dis 2010; 69:522-528.

12. Strangfield A, Hierse F, Rau R, Burmester GR, Krummel-Lorenz B, Demary W, et al. Risk of incidence or recurrent malignancies among patients with rheumatoid arthritis exposed to biologic therapy in the German biologics register RABBIT. Arthritis Res Ther 2010; 12:R5.

13. Strangfeld A, Herse F, Kekow J, von Hinueber U, Tony HP, Dockhorn R, et al. Comparative effectiveness of tumour necrosis alpha inhibitors in combination with either methotrexate or leflunomide. Ann Rheum Dis 2009; 68:1856-1862.

14. BIOBADASER. BIOBADASER: http://biobadaser. ser.es/biobadaser/eng/docs/report_260603.PDF

15. Gomez-Reino JJ, Carmona L, Descalzo MA. Risk of tuberculosis in patients treated with tumor necrosis factor antagonists due to incomplete prevention of reactivation of latent infection. Arthritis Rheum 2007; 57:756-761.

16. Ljung L, Rantapää-Dahlqvist S, Jacobsson LT, Askling J. Response to biological treatment and subsequent risk of coronary events in rheumatoid arthritis. Ann Rheum Dis. 2016 Mar 16. pii: annrheumdis-2015-208995. doi: 10.1136/ annrheumdis-2015-208995. [Epub ahead of print].

17. Pappas DA1, Oh C, Plenge RM, Kremer JM, Greenberg JD. Association of rheumatoid arthritis risk alleles with response to anti-TNF biologics: results from the CORRONA registry and metaanalysis. Inflammation. 2013 Apr; 36(2):279-84.

18. Yoshida K, Radner H, Mjaavatten MD, Greenberg JD, Kavanaugh A, et al. Incidence and Predictors of Biological Antirheumatic Drug Discontinuation Attempts among Patients with Rheumatoid Arthritis in Remission: A CORRONA and NinJa Collaborative Cohort Study. J Rheumatol. 2015 Dec; 42(12):2238-46.

19. Gerhold K, Richter A, Schneider M, Bergerhausen HJ, Demary W, Liebhaber A, et al. Health-related quality of life in patients with long-standing rheumatoid arthritis in the era of biologics: data from the German biologics register RABBIT. Rheumatology (Oxford). 2015 Oct; 54(10):1858-66.

20. Ajeganova S, Humphreys JH, Verheul MK, van Steenbergen HW, van Nies JA, Hafström I. Anticitrullinated protein antibodies and rheumatoid factor are associated with increased mortality but with different causes of death in patients with rheumatoid arthritis: a longitudinal study in three European cohorts. Ann Rheum Dis. 2016 Jan 12. pii: annrheumdis-2015-208579. [Epub ahead of print].

21. de la Vega M, Casado G, Citera G, Soriano E, Venarotti H, et al. Primer reporte de efectos adversos de tratamientos biológicos en Argentina. Informe de Registro BIOBADASAR. Rev Arg Reumatol 2011; 22:40-54.

22. de la Vega M, da Silveira de Carvalho H, Ventura Ríos L, Goycochea Robles M, Casado G. The importance of rheumatology biologic registries in Latin America. Rheumatol Int 2013; 33:827-835. 